\title{
O-Cam: A new paradigm for investigating the effects of ostracism
}

\author{
RANI GOODACRE AND LiSA ZADRO \\ University of Sydney, Sydney, New South Wales, Australia
}

\begin{abstract}
A new ostracism paradigm - O-Cam - was designed to combine the best qualities of both social ostracism (i.e., face-to-face interaction between the target and sources of ostracism) and cyber ostracism (i.e., confederatefree, highly controlled designs) paradigms. O-Cam consists of a simulated Web conference during which participants are either ostracized or included by 2 other participants whose actions, unbeknownst to the participants, are actually pretaped. The findings of preliminary studies indicate that $\mathrm{O}-\mathrm{Cam}$ provides a powerful ostracism experience that yields psychological and behavioral responses that are consistent with those in other ostracism paradigms (e.g., Cyberball; Williams, 2007). Moreover, unlike in many previous ostracism paradigms, O-Cam provides researchers with the flexibility to manipulate the physical appearance and the verbal/nonbehavior of the sources of ostracism without the need for confederates.
\end{abstract}

Ostracism - being excluded or ignored - is a powerful form of social punishment (Williams, 2001). Over the past decade, researchers have demonstrated that this social phenomenon is unique from other forms of interpersonal conflict in its ability to deplete four primary human needs: belongingness, control, self-esteem, and meaningful existence (Williams, 2007). In addition to adversely affecting primary needs, ostracism has been shown to have a host of behavioral consequences ranging from conformity (Williams, Cheung, \& Choi, 2000) to social loafing (Williams \& Sommer, 1997).

In order to assess the effects of ostracism, Williams and his colleagues developed several laboratory-based paradigms that can be broadly classified into two categories: (1) social or face-to-face ostracism paradigms, and (2) cyber paradigms. Social or face-to-face ostracism paradigms are those in which the target is ignored/excluded during an interaction that takes place in the physical presence of the source(s)/ostracizers - for instance, during a game of ball toss (see, e.g., Williams \& Sommer, 1997), a conversation (e.g., Ezrakhovich et al., 1998), or a role-play task (i.e., the train ride; Zadro, Williams, \& Richardson, 2005). Cyber ostracism paradigms are those in which the target is ostracized by the source(s) during an electronic exchange-for instance, a chat-room discussion (Williams et al., 2002), text messaging (Smith \& Williams, 2004), or an Internet game of ball toss (Cyberball; e.g., Williams et al., 2000).

Social paradigms boast high ecological validity, permitting the findings to be generalized to a variety of situations in which targets are ignored in the presence of sources (e.g., in the home, school, or workplace). However, social paradigms have been used in only a small number of ostracism studies. This is primarily due to the fact that these paradigms require confederates to play the part of the sources of ostracism; hence, they are rather resource intensive. Cyber paradigms, on the other hand, are simple to run, since they eliminate the need for confederates. For instance, in the cyber paradigm Cyberball (see Williams et al., 2000; Williams \& Jarvis, 2006), targets play a game of ball toss with two (computer-generated) players whose responses are preprogrammed. The confederate-free design of cyber paradigms ensures that these studies afford a great deal of control over the experiment. This is not the case with face-to-face paradigms, in which it is difficult to guarantee that the verbal and nonverbal behavior of confederates remains the same for each participant, even when confederates are extensively trained.

Overall, cyber paradigms, particularly Cyberball, have revolutionized the way in which ostracism research has been conducted, and they now dominate the existing body of ostracism literature. Yet, cyber paradigms do not always induce the same responses in targets as do social paradigms. Studies comparing the effects of social and cyber ostracism have reported similar patterns of primary needthreat after ostracism, irrespective of the paradigm; however, the behavioral responses of ostracized participants during the exclusion episode tend to differ as a function of the type of paradigm (Williams et al., 2002). For instance, targets who are ostracized face-to-face often become quiet and withdrawn; after a minute or so of ostracism, they tend to passively watch the sources without trying to confront them or reinstate themselves into the interaction (Williams et al., 2002). In contrast, targets who have been ostracized in cyber paradigms often attempt to reinclude themselves in the interaction by engaging in acts of ego assertion (e.g., yelling at the computer, talking to them-

L.Zadro, lisa.zadro@sydney.edu.au 
selves), or by challenging the sources about their exclusionary tactics (Williams, 2007; Williams et al., 2002).

The behavioral differences that may result from using these different paradigms, coupled with the fact that cyber paradigms are far more prevalent in the literature, suggest that our understanding of the psychological and behavioral consequences of social ostracism may not be as extensive as that of cyber ostracism. This is of particular concern, given that there is anecdotal and empirical evidence that social ostracism may lead to harmful behaviors directed toward the self(e.g., self-mutilation, suicidal ideation; Zadro, 2004), as well as toward sources and innocent bystanders (e.g., the destruction of personal property, the instigation of verbal or physical abuse; Zadro, 2004; see also Leary, Kowalski, Smith, \& Phillips, 2003; Leary, Twenge, \& Quinlivan, 2006; Warburton, Williams, \& Cairns, 2006).

Thus, our aim was to develop a new paradigm, O-Cam, that combined the positive qualities of both cyber and social ostracism paradigms - that is, high ecological validity, high experimental control, standardized presentation of confederates, and a simple method of administration (see Goodacre, 2007). O-Cam simulates a face-to-face ostracism experience (i.e., being ignored during a Web conference) while maintaining a highly controlled, confederatefree design.

\section{The O-Cam Paradigm}

The O-Cam paradigm is a simulated Web cam conference that takes place between a participant and 2 students from a local university (this aspect of the paradigm can be modified so that the 2 participants are from any institution). Although the paradigm has the appearance of a real Webbased interaction, the "students" are actually actors whose actions have been prerecorded. Two O-Cam conditions are prerecorded: one in which the students appear to listen to the participant as he or she makes a speech (the inclusion condition), and another in which the students appear to listen to the participant's speech for $15 \mathrm{sec}$ and then turn to each other and begin having a conversation, completely ignoring the participant (the ostracism condition). A demonstration of the paradigm can be seen at www.psych.usyd.edu.au/ research/ostracism/ (username, guest; password, Bach).

In all of our studies to date, male participants viewed a film of 2 male student actors, and female participants viewed a film of 2 female student actors to avoid possible confounding due to sex bias. However, the gender and appearance of the students can be varied according to the aims of the study. All versions of the paradigm were filmed using a JVC GZ-MG505AA Hard Disk Camcorder and a Rode Directional Video Condenser microphone. The tapes were played through MediaLab software, which was programmed so as to give the videos the appearance of a Web conference (see Figure 1). ${ }^{1}$

O-Cam procedure. When participants enter the laboratory, they are informed that they will be trialing a new Web-conference program (UniLink Conferencing) with 2 students from another university. The experimenter explains that in this conference, they will all give a short, prepared (and uninterrupted) speech about their experiences of university life. Each student is allocated a number that

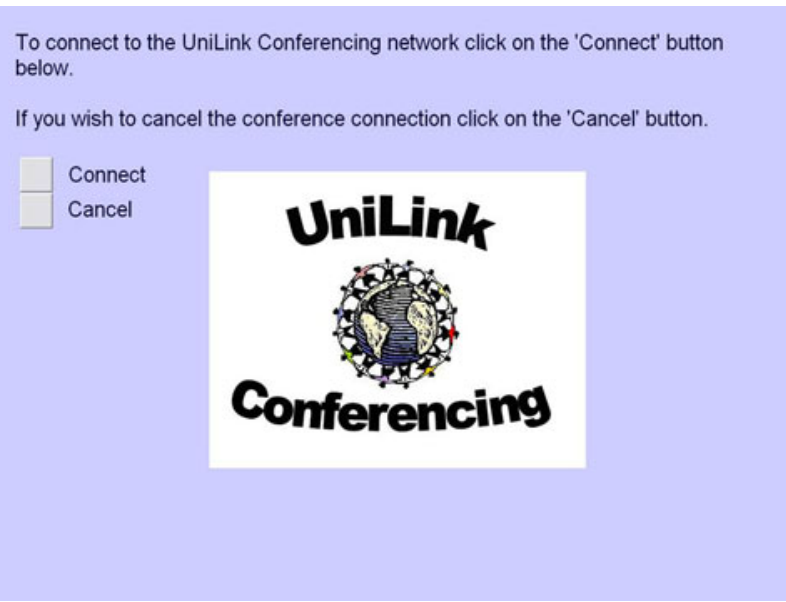

Figure 1. The "Connect to Conference" main page; playing the videos through MediaLab software enhances the Web conference cover story.

determines when he or she will speak during the conference; the participant is assigned the number " 3 ," signifying that he or she will be the third person to speak.

Participants receive a series of questions to assist them in preparing their speech (e.g., "Why did you decide to study at this University?"; "What are your majors?"; "What do you wish to do when you leave university?"). They are then given the opportunity to practice their speech with the experimenter in order to both reduce nervousness and ensure that they will talk for the full duration of the inclusion phase of the O-Cam film (approximately $1 \mathrm{~min}$ $30 \mathrm{sec}$ ). This practice is necessary in order to maintain the illusion of a real-time interaction.

When the participant has completed the speech, the experimenter leads the participant to the Web conference setup area (see Figure 2). Participants are seated at a computer with a camera. The camera is not a functional Web

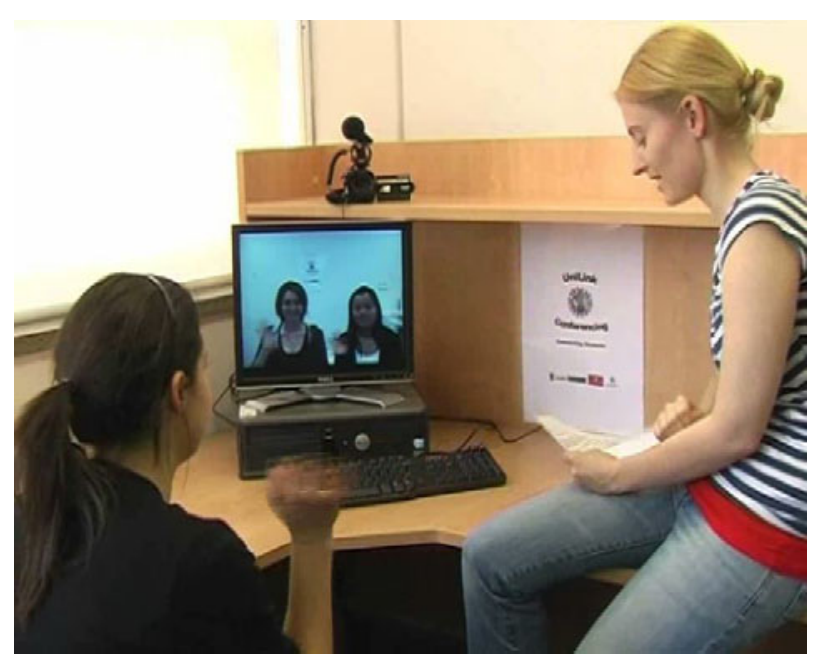

Figure 2. The experimenter introducing the participant to 2 female "students" at the beginning of the O-Cam conference film. Note that the participant is waving at the screen, indicating her belief that she is taking part in a real-time Web conference. 


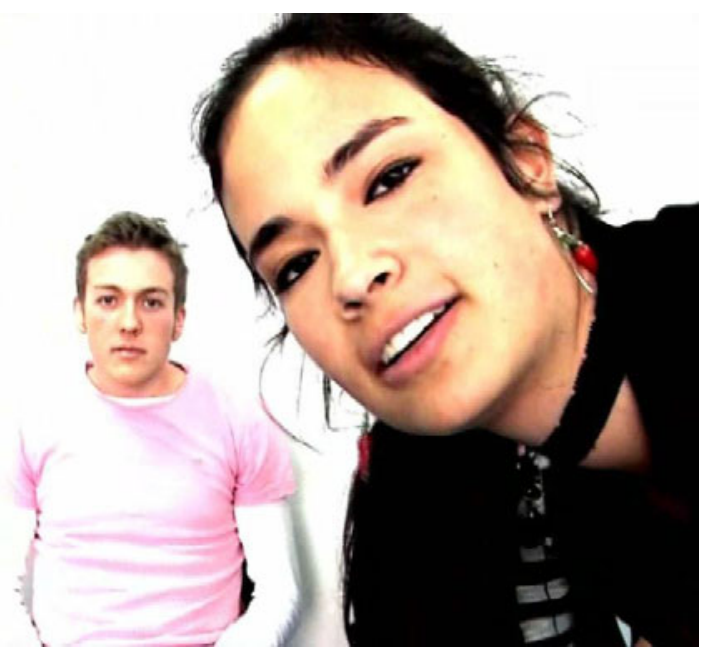

Figure 3. The "assistant" (an actor) helps to set up the conference during a scripted section of the O-Cam film, appearing to speak to the experimenter.

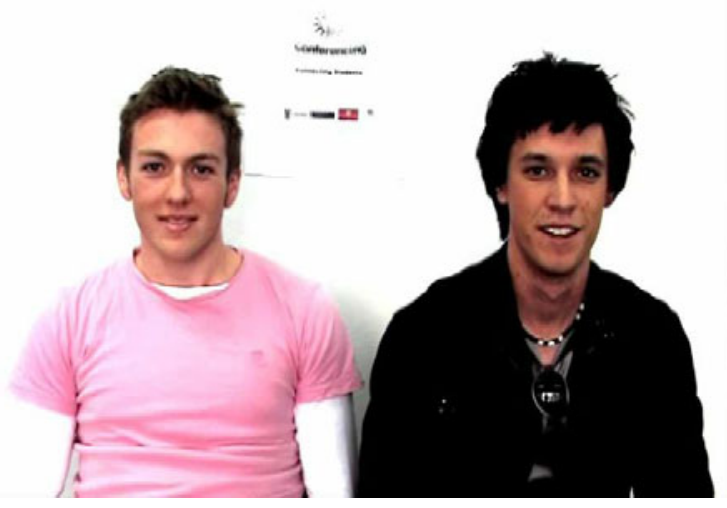

Figure 4. Two male "students" (who are actually actors) including/ attending to the participant during the participant's speech.

cam; rather, it is there to give the illusion of a Web conference and can be used to record participants' behavior during the conference, which can then be coded. ${ }^{2}$

Once the participants are seated, the experimenter informs the participants that they will begin the conference (i.e., they begin the appropriate prerecorded tape). On-screen, 2 students are seated next to each other, and an "assistant"- who is, in actual fact, another actor-is nearby to help set up the conference (see Figure 3). To add to the plausibility of the cover story, a series of scripted "interactions" between the experimenter, the assistant, and the 2 students are played out prior to the actual conference in order to convince the participant that these events are happening in real time. That is, the experimenter and assistant greet each other and work together to set the microphone levels on the computer; the experimenter bids farewell to the assistant, who leaves the room, and the experimenter instructs the students to move closer to the camera, with which the students comply (see the demonstration).

The experimenter then introduces each student by number - that is, Student 1, Student 2, and Student 3, the participant - and informs them that they will be giving their speeches in this order. The experimenter informs the participants that they are not to interrupt the speeches and that, once they have completed their turn, they are to indicate that they have finished so that the next person can begin his or her speech. The experimenter then states that he or she has to leave the room to set up for another conference and instructs the 3 students to begin after the experimenter has left the room. The films are timed to ensure that the scripted interactions flow seamlessly; Student 1 begins his or her talk as soon as the experimenter leaves the room, thereby creating the ruse of real interaction.

In each conference, the students talk for approximately $1.5 \mathrm{~min}$ each; then the real participant speaks. During the participant's speech, the ostracism manipulation takes place. There are two versions of the pretaped films: inclusion and ostracism. Participants in the inclusion condition are "attended to" by the 2 students (i.e., the students smile and appear to look at the participant) for the duration of their speech (1 min $30 \mathrm{sec}$; see Figure 4). In contrast, participants in the ostracism condition are "attended to" by the 2 students for approximately $15 \mathrm{sec}$; then the students begin to speak to each other and completely ignore the participant for the remainder of the participant's speech ( $1 \mathrm{~min} 15 \mathrm{sec}$; see Figure 5 and the demonstration). All of the participants are instructed that the conference will terminate after $10 \mathrm{~min}$; at the end of the inclusion or ostracism phase of the films, the assistant returns and interrupts the conference, stating, "Time is up!" and instructs the students to complete any postconference questionnaires/ tasks. The conference then ends.

Once the conference connection is terminated, participants can complete measures that assess their psychological and/or behavioral responses to ostracism. To as-

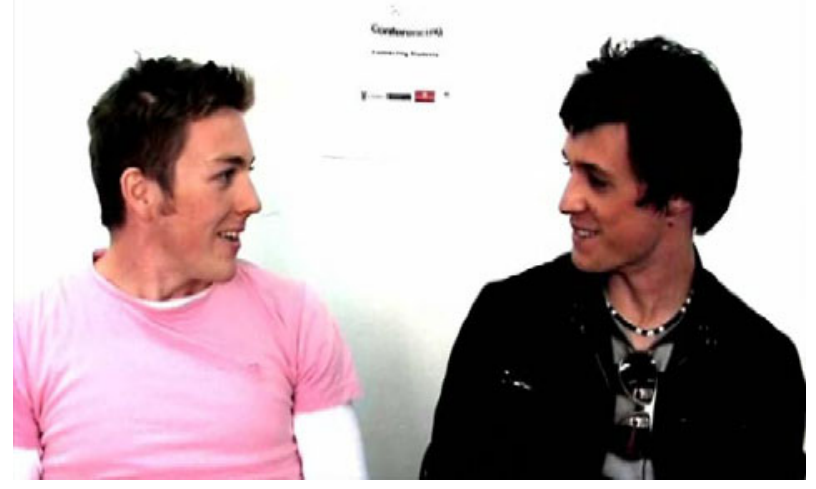

Figure 5. Two male "students" ostracizing/ignoring the participant during the participant's speech. 
sess psychological responses, we typically use standard measures of primary need-threat and mood (see Zadro, Boland, \& Richardson, 2006). We have also assessed a variety of postostracism responses, most notably aggression against the sources of ostracism and innocent bystanders (using the "revenge/retaliation" questionnaire devised by Goodacre, 2007).

After assessing behavioral responses, participants are fully debriefed. In all rejection and self-exclusion studies, every effort must be taken to ensure that the participants do not leave the laboratory in a distressed state. Considerable care should be taken by researchers to explain the ostracism manipulation and the necessity for deception in studies of this nature. In addition to course credit, we often give participants a treat to help elevate their mood (i.e., a choice between a chocolate bar and healthy snack).

\section{Assessing the Effectiveness of O-Cam}

One of the primary concerns when creating O-Cam was whether or not participants would believe the Web cam cover story. In this respect, the paradigm was successful. For instance, in one study of 55 first-year psychology students at the University of Sydney ( 25 male and 30 female; $M$ age $=20.07$ years, $S D=3.39$ ), all of the participants appeared to be convinced that the O-Cam conference was real (Goodacre, 2007). Both the included and ostracized participants interacted with the actors in the O-Cam films as if they were real people, smiling and nodding while listening to them speak. At the end of the conference, some participants even said "goodbye" to the students. The enthusiasm that was expressed by targets of inclusion during the conference was also evident in their responses to an open-ended manipulation check, which included statements such as "Being able to connect with someone at another uni is pretty cool" and "This conference as a first step to meet someone outside of your own social circle is an absolutely great concept which I recommend to be introduced to universities." Additional standard manipulation checks routinely used in ostracism research also revealed that O-Cam successfully induced feelings of being excluded and ignored.

Although we have administered several ostracism paradigms in the past (e.g., the train ride, Zadro et al., 2005; Cyberball, Zadro et al., 2006), we were struck by the realism and power of the O-Cam ostracism manipulation. Those in the ostracism condition began their turn to speak with enthusiasm equal to that of their included counterparts, clearly attempting to establish a favorable impression on the 2 students. However, their enthusiasm ended abruptly when they began to be ignored. The majority of ostracized targets initially appeared astonished and nervous, staring at the students as they began their conversation over the participant's speech. As the ostracism episode continued, participants became more self-conscious, slumping in their chairs, their facial expressions showing increased discomfort. By the end of the conference, they appeared withdrawn and dejected (for a comprehensive analysis of the nonverbal behavior displayed by ostracized participants during O-Cam, see Goodacre, 2007). A few of the participants attempted to regain the sources' atten- tion (i.e., by making verbal remarks or physical gestures); however, these gestures were not made easily. Rather, these participants appeared to need to build up their courage, and even then, most of these responses were made reluctantly (e.g., a softly spoken or mumbled "umm ... excuse me"). When these weak attempts to reclaim their turn to talk failed, the courage that these participants had mustered seemed to vanish instantly, and they became even more discomforted and dejected. In general, the behavior of targets during O-Cam best resembles the passive, withdrawn behavior of targets in face-to-face paradigms rather than the assertive, confrontational behavior of targets in cyber paradigms (Williams et al., 2002); this suggests that although this paradigm may be administered over a cyber medium, the physical "presence" of the two sources elicits the same behavioral responses as face-to-face episodes of exclusion. Clearly, however, further research is necessary to distinguish whether O-Cam has more in common with its face-to-face or cyber counterparts.

Thus, the O-Cam paradigm appeared to be successful in simulating a powerful, real-world ostracism experience.

Psychological and behavioral responses to $\mathrm{O}-\mathrm{Cam}$. In addition to providing a powerful and convincing ostracism experience, the O-Cam paradigm induces the same pattern of psychological outcomes - specifically, primary need-threat as assessed by a standard need-threat measure (see Zadro et al., 2006) - as do other established ostracism paradigms (e.g., Cyberball and the train ride; see Williams, 2007). For instance, Goodacre (2007) found that participants who were ostracized during O-Cam reported significantly greater need-threat $(M=30.46$, $S D=6.62)$ than did participants who had been included during O-Cam $(M=40.22, S D=6.83)[F(1,51)=34.26$, $\left.p<.0005, \eta_{\mathrm{p}}^{2}=.402\right] .^{3}$

In order to assess the delayed effects of ostracism on primary needs, Goodacre (2007) reassessed the primary needs of participants at the end of the testing session. In accordance with previous Cyberball research (e.g., Oaten, Jones, Williams, \& Zadro, 2008; Zadro et al., 2006), a significant interaction effect between inclusionary status (i.e., ostracism vs. inclusion) and time was found [Wilks's $\Lambda=.769, F(1,51)=15.35, p<.00005, \eta_{\mathrm{p}}^{2}=$ .231]. Follow-up analyses revealed that, at the delayed assessment, targets of ostracism $(M=42.18, S D=7.24)$ reported levels of primary needs similar to those reported by targets of inclusion $(M=44.30, S D=5.13)[F(1,53)=$ $1.56, p=.218]$. Thus, participants who were ostracized during $\mathrm{O}-\mathrm{Cam}$ regained their thwarted needs by the end of the study (i.e., $20 \mathrm{~min}$ later). Although future research will need to assess the longevity of the ostracism manipulation, these findings suggest that participants do not leave the laboratory suffering any lingering adverse effects of the O-Cam ostracism manipulation on their primary needsan essential finding for any ostracism paradigm.

The O-Cam paradigm not only thwarted primary needs, but also affected behavioral responses. Goodacre (2007) assessed the extent to which being ostracized during O-Cam led targets to endorse different forms of revenge and retaliation behaviors toward the sources of ostracism. Specifically, these forms of revenge and retaliation in- 
cluded active distancing (i.e., whether or not participants wished to have further contact with the sources of ostracism), reputation defamation (i.e., the extent to which participants would present the sources in an unfavorable light by stating that they would engage in unsavory acts such as lying, stealing, and cheating), and resource removal (i.e., the extent to which participants would allocate less reward money to sources of ostracism when the participant was asked to share a monetary pool of $\$ 100$ between him- or herself and the sources). As compared with those who had been included in the O-Cam conference, ostracized participants were more likely to distance themselves from the sources (ostracized participants, $M=2.71, S D=1.08$; included participants, $M=2.00, S D=0.679)[F(1,51)=$ $\left.8.71, p=.005, \eta_{\mathrm{p}}^{2}=.146\right]$, exhibit significantly higher rates of reputation defamation (ostracized participants, $M=91.25, S D=21.18$; included participants, $M=$ $74.19, S D=7.46)\left[F(1,51)=16.52, p<.0005, \eta_{\mathrm{p}}^{2}=\right.$ .245], and allocate significantly more of the total $\$ 100$ pool to themselves rather than share it equally with the sources (ostracized participants, $M=\$ 46.43, S D=$ 24.23 ; included participants, $M=\$ 32.90, S D=2.56$ ) $\left[F(1,51)=8.95, p=.004, \eta_{\mathrm{p}}^{2}=.149\right] .{ }^{4}$

Thus, after being ostracized during O-Cam, participants were willing, when presented with the opportunity, to engage in mild antisocial and vengeful behaviors toward those who had ostracized them. This supports the findings in the rejection literature, whereby targets of rejection act less charitably, are less likely to offer assistance (Twenge, Baumeister, DeWall, Ciarocco, \& Bartels, 2007), and are more likely to give others negative evaluations (Twenge, Baumeister, Tice, \& Stucke, 2001) than those who were not rejected. These results, however, are not typical in the ostracism literature. Specifically, targets of ostracism usually behave prosocially after being ostracized during Cyberball (see, e.g., Williams et al., 2000) and behave aggressively in face-to-face ostracism paradigms only if their sense of control is further thwarted (Warburton et al., 2006). However, prior ostracism research has typically focused on assessing aggressive responses to innocent third parties after exclusion (e.g., Warburton et al., 2006). In the O-Cam study, targets were given the opportunity to seek vengeance against those who had ignored them, which may have elicited heightened aggressive tendencies in targets. Research is currently under way to determine whether the vengeful and retaliatory responses induced by $\mathrm{O}-\mathrm{Cam}$ are a function of the paradigm or of the fact that these behaviors were aimed toward sources rather than toward third parties.

Overall, O-Cam replicated and expanded the findings derived from other ostracism paradigms, thereby lending further support to its effectiveness as an ostracism paradigm.

\section{Distinguishing O-Cam From \\ Social Rejection Paradigms}

As an ostracism paradigm, O-Cam is unique in that it allows targets to be excluded in the physical presence of sources, yet does not require confederates. However, $\mathrm{O}-\mathrm{Cam}$ can also be distinguished from paradigms that ma- nipulate rejection. Rejection is typically operationalized as "a declaration by an individual or group that they do not (or no longer) want to interact or be in the company of the individual" (Williams, 2007, p. 429; for a discussion on the differences between ostracism and rejection and their propensity to lead to aggression, see Leary et al., 2006). Ostracism differs from rejection because it does not require sources to explicitly declare their intent to ignore the target; instead, participants are excluded and ignored, which is what occurs in the O-Cam paradigm. In rejection paradigms, the target is typically informed - usually by the experimenter or by false feedback of some form - that they have been excluded by other group members from a subsequent task or interaction (Buckley, Winkel, \& Leary, 2004; Stillman, Baumeister, Lambert, \& Crescioni, 2009; Twenge et al., 2001). For instance, in the "get acquainted" paradigm (Twenge et al., 2001), targets interact with a small group of participants in the getting-to-know-you exercise. After the task, members of the group are physically separated and asked to nominate the 2 people from the group with whom they would like to perform a subsequent task. The target then receives feedback from the experimenter that is either rejecting (i.e., they are informed that no one in the group wished to work with them on a subsequent task) or inclusionary/accepting (i.e., they are informed that everyone in the group wanted to work with them on a subsequent task). In another rejection paradigm, Buckley et al. manipulated rejection via computerized false feedback. Participants are assigned to be "speakers" and are instructed to answer questions about themselves while an "evaluator" in another room (actually a confederate) gives them moment-to-moment feedback on the extent to which they wish to get to know the speaker. This feedback is delivered via a panel of numbered squares on the participant's computer screen and varies from constant acceptance to constant rejection.

$\mathrm{O}-\mathrm{Cam}$ is unique among such rejection paradigms because it allows the target to be ignored while in the visual presence of sources. Moreover, it does not require the participant to complete a prior task to justify the exclusion (e.g., the group task in the get-acquainted paradigm; Twenge et al., 2001). The exclusion in O-Cam is immediate and delivered by the sources; it does not require the rejection manipulation to be delivered via false feedback by the experimenter or a questionnaire.

It would be possible, however, to modify O-Cam so that it resembles a rejection paradigm. For instance, during their pretaped speeches, the confederates could explicitly state that they did not wish to interact with the target on a subsequent task. This would allow the sources to present the negative/rejecting feedback rather than have it delivered by the experimenter or via false feedback. Future researchers may wish to investigate whether being explicitly rejected by the sources leads to a different pattern of psychological and behavioral effects than does simply being ignored by the sources without any prior rejection. From a methodological perspective, researchers may also be interested in exploring whether rejection leads to the same consequences when it is delivered by the source(s) as when it is delivered by the experimenter or through false feedback 
(e.g., being informed about the rejection by a third party may buffer some of the aversive consequences).

\section{Current and Future Research Using O-Cam}

The innovative experimental design of O-Cam is gathering considerable interest from those in the field since it gives ostracism researchers the opportunity to broaden the scope of their investigations, particularly if they wish to examine aspects of ostracism that require face-to-face interaction with the sources. There are several O-Cam studies that are currently under way. One area currently under investigation is how the identity of the sources influences the effects of ostracism on targets. Although previous cyber studies have manipulated aspects of source identity through "source profiles" that are revealed to the participant by the experimenter during the introductory instructions (e.g., the political affiliation of the sources, Gonsalkorale \& Williams, 2007; and computer preference, Mac or PC, Williams et al., 2000), the O-Cam paradigm can manipulate aspects of source identity through visual elements or the content of the sources' speeches. In fact, several researchers are currently using the O-Cam to conduct studies that examine how the physical features of sources (e.g., age, gender, attractiveness, race), as well as the content of the sources' speech (e.g., whether they express negative comments about the target's racial group) influence the effects of ostracism on targets. One such program of research is currently examining the link between ostracism by ethnic outgroups and racially motivated violence.

The O-Cam paradigm could also be modified to examine specific aspects of the ostracism experience as elucidated by Williams's (2001) model of ostracism. For instance, O-Cam has been used to assess only complete ostracism; that is, sources attend to the target briefly and then ignore him or her for the remainder of the conference by withholding all verbal and nonverbal communication. However, the procedure could be modified to examine the effects of partial ostracism; that is, the sources could glance at the target at intervals, thereby giving the target the impression that he or she is being attended to. Researchers could then compare the effects of complete and partial ostracism on a range of psychological and behavioral measures. The quantity of ostracism experienced by targets could also be varied by increasing or decreasing the proportion of time that the target is initially attended to by the sources and then subsequently ignored. Similar modifications to the tapes and procedure would allow researchers to manipulate other aspects of the ostracism experience, such as the sources' motives or the causal clarity of the ostracism episode. Such modifications would allow ostracism researchers the opportunity to determine how specific elements of the exclusion experience ameliorate or exacerbate its aversiveness.

Other researchers are using O-Cam to investigate the effects of ostracism on specific samples. Some are using $\mathrm{O}-\mathrm{Cam}$ to assess the effects of ostracism on children, since $\mathrm{O}-\mathrm{Cam}$ tends to be more visually engaging than the Cyberball paradigm. Clinical psychologists are currently using $\mathrm{O}$-Cam to assess the effects of ostracism on socially anx- ious and socially phobic samples; they have chosen to use $\mathrm{O}-\mathrm{Cam}$ rather than other paradigms because it simulates real-life episodes of ostracism feared by socially anxious/ phobic individuals. Finally, we are currently modifying the O-Cam paradigm so that it can be used to investigate the effects of ostracism on sources (i.e., ostracizers) - an area that has received little empirical investigation.

Overall, O-Cam is an effective ostracism paradigm that combines the experience of social ostracism with the convenience and procedural control of cyber paradigms. Accordingly, it has the potential to be a valuable tool in investigating this complex phenomenon.

\section{AUTHOR NOTE}

This research was funded by ARC Discover Project Grant DP0666929 held by L.Z. (coinvestigator Michelle Moulds). The authors thank Guy Chapman, Suzanne Ly, Anna Ferdman, Susy Natal, Tim McGill, Michael Noetel, Jennifer Follers, Phillip Goodacre, and Aliza Werner-Seidler for their assistance in creating the O-Cam materials. We also thank the Editor and our three anonymous reviewers for their excellent advice and suggestions. Address all correspondence to L. Zadro, School of Psychology, University of Sydney, Sydney 2006, Australia (e-mail: lisa.zadro@ sydney.edu.au).

\section{REFERENCES}

Buckley, K. E., Winkel, R. E., \& Leary, M. R. (2004). Reactions to acceptance and rejection: Effects of level and sequence of relationship evaluation. Journal of Experimental Social Psychology, 40, 14-28. doi:10.1016/S0022-1031(03)00064-7

Ezrakhovich, A., Kerr, A., Cheung, S., Elliot, K., Jerrems, A., \& Williams, K. D. (1998, April). Effects of norm violation and ostracism on working with the group. Paper presented at the Society of Australasian Social Psychologists, Christchurch, New Zealand.

Gonsalkorale, K., \& Williams, K. D. (2007). The KKK won't let me play: Ostracism even by a despised outgroup hurts. European Journal of Social Psychology, 37, 1176-1186. doi:10.1002/ejsp.392

GOODACRE, R. (2007). O-Cam: A new social ostracism paradigm. Unpublished honors thesis, University of Sydney.

Leary, M. R., Kowalski, R. M., Smith, L., \& Phillips, S. (2003). Teasing, rejection, and violence: Case studies of the school shootings. Aggressive Behavior, 29, 202-214. doi:10.1002/ab.10061

Leary, M. R., Twenge, J. M., \& Quinlivan, E. (2006). Interpersonal rejection as a determinant of anger and aggression. Personality \& Social Psychology Review, 10, 111-132. doi:10.1207/s15327957pspr1002_2

Oaten, M., Jones, A., Williams, K. D., \& Zadro, L. (2008).The effects of ostracism on self-regulation in the socially anxious. Journal of Clinical \& Social Psychology, 27, 471-504. doi:10.1521/ jscp.2008.27.5.471

Smith, M. A., \& Williams, K. D. (2004). R U There? Ostracism by cell phone text messages. Group Dynamics: Theory, Research \& Practice, 4, 291-301. doi:10.1037/1089-2699.8.4.291

Stillman, T. F., Baumeister, R. F., Lambert, N. M., \& Crescioni, A. W. (2009). Alone and without purpose: Life loses meaning following social exclusion. Journal of Experimental Social Psychology, 45, 686-694. doi:10.1016/j.jesp.2009.03.007

Twenge, J. M., Baumeister, R. F., DeWall, C. N., Ciarocco, N. J., \& BARTEls, J. M. (2007). Social exclusion decreases prosocial behavior. Journal of Personality \& Social Psychology, 92, 56-66. doi:10.1037/0022-3514.92.1.56

Twenge, J. M., Baumeister, R. F., Tice, D. M., \& Stucke, T. S. (2001). If you can't join them beat them: Effects of social exclusion on aggressive behavior. Journal of Personality \& Social Psychology, 6, 10581069. doi:10.1037/0022-3514.81.6.1058

Warburton, W. A., Williams, K. D., \& Cairns, D. R. (2006). When ostracism leads to aggression: The moderating effects of control deprivation. Journal of Experimental Social Psychology, 42, 213-220. doi:10.1016/j.jesp.2005.03.005 
WiLliams, K. D. (2001). The power of silence. New York: Guilford.

Williams, K. D. (2007). Ostracism. Annual Review of Psychology, 58, 425-452. doi:10.1146/annurev.psych.58.110405.085641

Williams, K. D., Cheung, K. T., \& ChOI, W. (2000). Cyberostracism: Effects of being ignored over the Internet. Journal of Personality \& Social Psychology, 79, 748-762. doi:10.1037/0022-3514.79.5.748

Williams, K. D., Govan, C. L., Croker, V., Tynan, D., CruickSHANK, M., \& LAM, A. (2002). Investigations into differences between social- and cyberostracism. Group Dynamics: Theory, Research \& Practice, 6, 65-77. doi:10.1037/1089-2699.6.1.65

Williams, K. D., \& JARVIS, B. (2006). Cyberball: A program for use in research on ostracism and interpersonal acceptance. Behavior Research Methods, Instruments, \& Computers, 38, 174-180.

Williams, K. D., \& Sommer, K. L. (1997). Social ostracism by coworkers: Does rejection lead to loafing or compensation? Personality \& Social Psychology Bulletin, 23, 693-706. doi:10.1177/0146167297237003

ZADRO, L. (2004). Ostracism: Empirical studies inspired by real-world experiences of silence and exclusion. Unpublished doctoral dissertation, University of New South Wales.

Zadro, L., Boland, C., \& Richardson, R. (2006). How long does it last? The persistence of the effects of ostracism in the socially anxious. Journal of Experimental Social Psychology, 42, 692-697. doi:10.1016/j.jesp.2005.10.007

Zadro, L., Williams, K. D., \& Richardson, R. (2005). Riding the "O" train: Comparing the effects of ostracism and verbal dispute to targets and sources. Group Processes \& Intergroup Relations, 8, 125-143. doi: $10.1177 / 1368430205051062$

\section{NOTES}

1. A copy of the tapes may be obtained by writing to L.Z

2. This research paradigm was carefully reviewed by the University of Sydney Human Ethics Research Committee before it was given approval. Participants were required to give their consent to be filmed prior to the start of the study. At the end of the study, participants were informed that their behavior during the experiment would be analyzed, and they were given the opportunity to revoke their consent.

3. In accordance with previous ostracism research (see Zadro et al., 2006), a total overall need score was calculated, whereby belongingness, control, self-esteem, and meaningful existence items were reverse scored where necessary and then summed together. A lower score indicates greater need-threat; Cronbach alpha for Goodacre $(2007)=.86$. Scores for need-threat, both immediately after ostracism and after a delay, ranged from 12 to 60 .

4. Active distancing scores on each item ranged from 2 to 10 , with higher scores indicating that participants felt a greater desire to distance themselves from the 2 students in the O-Cam conference. Reputation defamation scores ranged from 64 to 160, with higher scores indicating higher levels of reputation defamation. Resource removal scores ranged from $\$ 0$ to $\$ 100$, with higher scores indicating that participants allocated more money to themselves rather than to the sources.

(Manuscript received September 25, 2009; revision accepted for publication April 6, 2010.) 